\title{
The impact of the dark matter-gas interaction on the collapse behavior of spherical symmetric systems
}

\author{
J. S. Klar and J. P. Mücket
}

\author{
Astrophysikalisches Institut Potsdam, An der Sternwarte 16, 14482 Potsdam, Germany \\ e-mail: [jklar; jpmuecket]@aip.de
}

Received 26 February 2008 / Accepted 4 April 2008

\begin{abstract}
Context. If the gas in the evolving cosmic halos is dissipating energy (cooling), then the dark matter (DM) halo also undergoes a compactification because of the variation in the gravitational potential. This is well-known as adiabatic contraction (AC).

Aims. Complementary to the AC, we investigate the resulting dynamical behavior of the whole system if the backreaction of the AC of DM onto the gas is taken into account.

Methods. Also to achieve sufficient high resolution within the central halo region, we use a crude fluid approximation for the DM obeying the adiabatic contraction behavior. Furthermore, we restrict ourselves to spherical symmetry and vanishing angular momentum of the studied matter configurations. The computations are done using a first-order Godunov scheme.

Results. Our results show that the dynamical interaction between gas and DM may lead to significantly shorter collapse times. If the gas cools, the dynamical behavior of the whole system depends strongly on the shape of the initial density profile.

Conclusions. Our findings indicate that, for a certain mass range of halo configurations, the dynamical interaction between gas and DM might be important for the halo evolution so it must be taken into account.
\end{abstract}

Key words. cosmology: dark matter - Galaxy: formation

\section{Introduction}

During the past decade, considerable progress has been made toward understanding the structure formation process in the universe. In particular, the dynamics and evolution of the dissipationless component, i.e. the formation and evolution of the dark matter (DM) halos, has been studied in great detail. The outcome of all the cosmological simulations is that the DM component forms a nearly stable halo with an almost universal density shape, which is approximated well by the profile fit given by Navarro et al. (1997) (NFW profile).

However, the density profiles derived from the observed rotation curves of galaxies (in particular, from LSB galaxies) show much shallower profiles than the NFW profile or even indicate a core structure (Marchesini et al. 2002; Gentile et al. 2004; Kuzio et al. 2006; Salucci et al. 2007). Therefore, the question arises whether the DM halo profiles really have a universal shape and, in particular, which mechanisms or processes could then influence or change the DM density profile towards the observed ones. The structure of the inner DM density profile (cuspy or core like) is important with respect to various processes within the innermost parts of galaxies and clusters of galaxies. The galaxy-galaxy lensing is a very promising observational method of mapping the DM distribution in galaxy halos. For a successful reconstruction, the knowledge of the density profile for a single DM halo is necessary, however (Kleinheinrich et al. 2006). The innermost DM density profile, i.e. how much concentrated the DM is within the central parts, is of particular interest if considering the consequences of possible DM annihilation and estimating the $\gamma$-ray luminosity of Galactic halos (see, e.g., Stoehr et al. 2003; Ascasibar et al. 2006; Diemand et al. 2007).
In contrast to the $\mathrm{DM}$, the baryonic fraction, $\left(M_{\mathrm{b}} \approx\right.$ $\left.0.2 M_{\mathrm{DM}}\right)$ is able to dissipate energy, and may cool, and eventually form a configuration that is much more concentrated than the surrounding DM. Due to the gravitational coupling of both components the DM responds dynamically on the compactification of the baryonic gas, and the density shape of the DM changes. That effect was first studied by Eggen et al. (1962) using the model of adiabatic contraction (AC). Zeldovich et al. (1980) used the approach of adiabatic invariants to consider an analogous situation with respect to mass constraints on Leptons. A well-elaborated form of the AC model was given by Blumenthal et al. (1986). The model was later investigated and tested by Ryden \& Gunn (1987), Cardone \& Sereno (2005), Sellwood \& McGaugh (2005), Vasiliev (2006), Dutton et al. (2007) and has been partly modified by Jesseit et al. (2002). In particular, it has been considered within a cosmological context by Gnedin et al. (2004). From the above studies, it is not clear, however, which processes mainly influence the final DM profile. Gnedin et al. (2004) have investigated how mergers and different cooling periods for the baryonic gas act on the DM dynamics. However, within mergers, mainly the interaction of the DM particles is important. In the very inner region the steepest matter distribution defines the DM profile. The formation of the final DM profile might depend on the relation of cooling and dynamical times. The AC model assumes a relatively slow (adiabatic) changing of the potential. Since the convergence of the simulations within $r \ll r_{\text {Virial }}$ is still not proven, it is difficult to come to a final conclusion about the density evolution in the central region. However, this is the region that is most affected by any concentration of the gas as the result of cooling processes. 
We want to consider the relatively artificial situation of a spherically symmetric matter distribution with fixed masses and limited spatial extension. The mass relation of gas to DM is fixed as 0.25 . This ratio on average varies for the observed cosmic structures (galaxies, clusters, dwarf galaxies, etc.) since it depends on the particular conditions of their formation. It is also correlated with the total mass of objects. Thus, the assumed unique mass ratio of about 0.25 is taken in our considerations as an arbitrary value. To explore the dynamical interaction between the DM and gas we start from a stable stationary configuration and then let the gas cool. Thus, no effects of particular matter infall or merger-like mass growth is considered. To enhance the resolution considerably, we use a crude gas/fluid approximation for the description of the DM assuming that the violent relaxation processes are fast enough to satisfy a nearly Maxwellian velocity distribution of the DM particles each time and within each shell, provided a sufficiently coarse grained shell distribution is considered. A similar treatment of the DM was used before in Chièze et al. (1997) and Teyssier et al. (1997). Therefore, we describe with accuracy the interaction of two gases/fluids, which interact only gravitationally, and only the gas component is able to dissipate/cool. This allows us to consider not only the dynamical response of the DM on the baryonic cooling as described by the AC models but also the back-reaction on the gas dynamics.

Recently, Conroy \& Ostriker (2007) have investigated which kind of energy input processes in a system comparable to clusters of galaxies may prevent the cooling catastrophe. The outcome is that the common action of various heating processes may be able to stabilize the system over cosmic time. However, a fine tuning is necessary, and the whole system is very sensitive to the parameters. They considered the dynamics of the gas assuming the DM potential to be static. Our studies demonstrate that looking at the gravitational interaction between gas and DM increases the tendency to an onset of the cooling catastrophe. The paper is organized as follows. In the next section we introduce our basic assumptions and give the main equations for the considered system. The numerical treatment is given in Sect. 3 . Section 4 contains the results for our various cases where the time evolution of the DM halo+gas system is followed if the gas cools. A discussion and summary of these results are given in Sect. 5. For better systematics, the details of the chosen numerical algorithm are described in the Appendix.

\section{Basic equations}

This section reviews our main assumptions and the basic equations describing the system of a DM halo with embedded gas.

\subsection{Dynamics of the baryonic gas}

The baryonic fraction of the halo is described as an ideal gas. The hydrodynamical equations for the density $\rho_{\mathrm{G}}$, the radial momentum density $\rho_{\mathrm{G}} u_{\mathrm{G}}$, and the energy density $\rho_{\mathrm{G}} \varepsilon_{\mathrm{G}}=\rho_{\mathrm{G}} u_{\mathrm{G}}^{2} / 2+\varepsilon_{\mathrm{G}, T}$ of a gravitationally bound gas sphere (we assume spherical symmetry) are

$$
\begin{aligned}
& \frac{\partial}{\partial t} \rho_{\mathrm{G}}+\frac{1}{r^{2}} \frac{\partial}{\partial r}\left[r^{2} \rho_{\mathrm{G}} u_{\mathrm{G}}\right]=0 \\
& \frac{\partial}{\partial t}\left(\rho_{\mathrm{G}} u_{\mathrm{G}}\right)+\frac{1}{r^{2}} \frac{\partial}{\partial r}\left[r^{2} \rho_{\mathrm{G}} u_{\mathrm{G}}^{2}\right]+\frac{\partial p_{\mathrm{G}}}{\partial r}=-\rho_{\mathrm{G}} \frac{\partial \Phi}{\partial r} \\
& \frac{\partial}{\partial t}\left(\rho_{\mathrm{G}} \varepsilon_{\mathrm{G}}\right)+\frac{1}{r^{2}} \frac{\partial}{\partial r}\left[r^{2} u_{\mathrm{G}}\left(\rho_{\mathrm{G}} \varepsilon_{\mathrm{G}}+p_{\mathrm{G}}\right)\right]=-\rho_{\mathrm{G}} u_{\mathrm{G}} \frac{\partial \Phi}{\partial r}-\Lambda
\end{aligned}
$$

with the gravitational potential $\Phi$ given by the Poisson equation and the cooling function $\Lambda$. Pressure $p$, internal energy $\varepsilon_{\mathrm{G}}$, and density $\rho_{\mathrm{G}}$ are related to each other by the polytropic equation of state:

$\varepsilon_{\mathrm{G}, T}=\frac{p_{\mathrm{G}}}{(\gamma-1) \rho_{\mathrm{G}}}$.

The gravitational potential is determined by the density distributions of the gas $\rho_{\mathrm{G}}$ and of the $\mathrm{DM} \rho_{\mathrm{DM}}$ via the Poisson equation:

$\frac{1}{r^{2}} \frac{\partial}{\partial r}\left[r^{2} \frac{\partial \Phi}{\partial r}\right]=4 \pi G\left[\rho_{\mathrm{G}}+\rho_{\mathrm{DM}}\right]$

To consider typical energy dissipation in the gas we assume cooling by recombination as given in Black (1981). We further assume that the gas consists of hydrogen, only. In that case we have

$$
\begin{aligned}
\Lambda= & 2.85 \times 10^{-27} T^{1 / 2} \\
& \times\left(5.914-0.5 \ln T+0.01184 T^{1 / 3}\right) n_{\mathrm{e}} n_{\mathrm{H}^{+}}
\end{aligned}
$$

where $n_{\mathrm{e}}, n_{\mathrm{H}^{+}}$denote the number densities of electrons and protons and $T$ the temperature. We consider only collisional ionization and suppose ionization equilibrium

$\Gamma_{\mathrm{EH}}(T) n_{\mathrm{H}} n_{\mathrm{e}}=\alpha_{\mathrm{H}}(T) n_{\mathrm{H}^{+}} n_{\mathrm{e}}$

where $\Gamma_{\mathrm{EH}}(T)$ and $\alpha_{\mathrm{H}}(T)$ is the corresponding ionization and recombination rate, respectively (Black 1981).

Since the gas is assumed to consist only of hydrogen and because of electric charge conservation, the relation between the number densities $n_{\mathrm{e}}, n_{\mathrm{H}^{+}}$, and $n=\rho_{\mathrm{G}} / m_{\mathrm{H}}$ can be determined straightforwardly as

$n_{\mathrm{e}}=n_{\mathrm{H}^{+}}=n \frac{\Gamma_{\mathrm{EH}}}{\Gamma_{\mathrm{EH}}+\alpha_{\mathrm{H}}}$,

and (4) can be written solely in terms of $n$ and $T$.

To simplify the calculations, we restrict ourselves to the above cooling mechanism throughout. One should be aware, however, that within large mass systems like galaxies and clusters of galaxies the dominant cooling processes are helium and heavy element line cooling and Bremsstrahlung. In a realistic treatment, the cooling function is a composite of the various contributions, the importance of which depends on the precise temperature and density conditions at each moment for the considered volume.

\subsection{The DM dynamics - a fluid approach}

The dynamics of DM is given by the collissionless Boltzmannequation for the one-particle phase-space density $f(\boldsymbol{x}, \boldsymbol{v}, t)$

$\frac{\partial f}{\partial t}+\sum_{i} v_{i} \frac{\partial f}{\partial x_{i}}+\sum_{i} \frac{\partial \Phi}{\partial x_{i}} \frac{\partial f}{\partial v_{i}}=0$

along with the Poisson equation for the mean gravitational potential $\Phi$ (see Eq. (3))

To obtain fluid-like equations of motion for DM, we computed the moments with respect to the velocities $v_{i}$ up to the second order using the $\mathrm{CBE}$ as described in Binney \& Tremaine (1988) and take into account that the velocities vanish at $r \rightarrow \infty$. Our main approximation is to assume that the $3 \mathrm{rd}$ order moments vanish. In particular, this is true if the velocity distribution is locally Maxwellian. Furthermore, we assume the velocity dispersion to be isotropic. Under these crude assumptions, one gets 
the system of equations to close. In addition to the Jeans equation, we then get an energy equation. If we formally assign the quantity

$p_{\mathrm{DM}}=\frac{1}{3} \rho_{\mathrm{DM}} \sigma_{\mathrm{DM}}^{2}=\rho_{\mathrm{DM}} \sigma_{\mathrm{DM}, r}^{2}$

to a pressure, then we have a full analogy to the gas equations. By making such an assumption we seem to suppose a kind of collision between the DM particles. However, if considering sufficiently coarse grained shell distributions for halos in N-body simulations, then one observes nearly Maxwellian velocity distributions within each mass shell (see, e.g. Hoeft et al. 2004). This is most probably the result of sufficiently fast violent relaxation during the collapse of the considered systems. In other words, the mechanism of violent relaxation (Lyndon-Bell 1967; Kull et al. 1997) has to redistribute the velocities of the DM faster then the dynamical timescale. For the initial configuration, this process of relaxation leading to some equilibrium and to corresponding velocity distribution is assumed to be completed at the moment $t=0$. Further on, the time-dependent evolution happens slowly enough that shell crossing is negligible.

We want to use that approximation to study the interplay between the gas and DM dynamics if energy dissipation in the gas happens. After transformation to spherical coordinates and restricting ourselves to isotropic velocity dispersion (i.e., $\beta=0$ ), we obtain the following system of equations:

$$
\begin{aligned}
& \frac{\partial}{\partial t} \rho_{\mathrm{DM}}+\frac{1}{r^{2}} \frac{\partial}{\partial r}\left[r^{2} \rho_{\mathrm{DM}} u_{\mathrm{DM}}\right]=0 \\
& \frac{\partial}{\partial t}\left(\rho_{\mathrm{DM}} u_{\mathrm{DM}}\right)+\frac{1}{r^{2}} \frac{\partial}{\partial r}\left[r^{2} \rho_{\mathrm{DM}} u_{\mathrm{DM}}^{2}\right]+\frac{\partial}{\partial r} \frac{\rho_{\mathrm{DM}} \sigma_{\mathrm{DM}}^{2}}{3}= \\
& -\rho_{\mathrm{DM}} \frac{\partial \Phi}{\partial r} \\
& \frac{\partial}{\partial t}\left(\rho_{\mathrm{DM}} \varepsilon_{\mathrm{DM}}\right)+\frac{1}{r^{2}} \frac{\partial}{\partial r}\left[r^{2} u_{\mathrm{DM}}\left(\rho_{\mathrm{DM}} \varepsilon_{\mathrm{DM}}+\frac{\rho_{\mathrm{DM}} \sigma_{\mathrm{DM}}^{2}}{3}\right)\right]= \\
& -\rho_{\mathrm{DM}} u_{\mathrm{DM}} \frac{\partial \Phi}{\partial r},
\end{aligned}
$$

with the total energy density $\rho_{\mathrm{DM}} \varepsilon_{\mathrm{DM}}=\rho_{\mathrm{DM}} v_{r}^{2} / 2+3 \rho_{\mathrm{DM}} \sigma_{r}^{2} / 2$.

Equations (9) together with the Poisson equation, provide a closed system for the dynamics of DM. As mentioned above, these equations offer a strong analogy to the hydrodynamic equations as described before. Using the continuity equation we can perform the energy equation in Eq. (9) to

$\frac{\mathrm{D}}{\mathrm{D} t} \log \left(\frac{\rho_{\mathrm{DM}}}{\sigma_{\mathrm{DM}}^{3}}\right)=\left[\frac{\partial}{\partial t}+u_{\mathrm{DM}} \frac{\partial}{\partial r}\right] \log \left(\frac{\rho_{\mathrm{DM}}}{\sigma_{\mathrm{DM}}^{3}}\right)=0$.

The quantity $\tau=\rho_{\mathrm{DM}} / \sigma_{\mathrm{DM}}^{3}$ represents the phase space density (see Taylor \& Navarro 2001) and the logarithm of $\tau$ is a measure for the entropy. Equation (10) states that the entropy is constant along the flux lines, and therefore also $\tau=$ const. Thus, the assumptions on the velocity distribution of the DM yields an effective equation of state (e.o.s.) that describes the process of adiabatic contraction of the DM sufficiently well. For the pressure analog $p_{\mathrm{DM}}=\rho_{\mathrm{DM}} \sigma_{\mathrm{DM}}^{2}$ we then get the relation $p_{\mathrm{DM}} \propto \rho_{\mathrm{DM}}^{5 / 3}$ which is formally identical to the adiabatic e.o.s. for an ideal gas.

For the static case, the Eq. (10) is identically fulfilled, and neither any restrictions on the e.o.s. nor any explicit expression for the e.o.s. can be obtained.

\section{Numerical calculations}

\subsection{The initial configuration of the DM-gas system}

For the initial distribution we choose a polytropic gas sphere (see Binney \& Tremaine 1988) of finite extension. We combine this with:

1. a spherical DM distribution obeying an effective e.o.s. with $\gamma_{\mathrm{DM}}=5 / 3$;

2. a DM distribution following an NFW-profile.

According to the previous findings, for the DM and for the gas may be assumed that

$p_{\mathrm{DM}}=\kappa_{\mathrm{DM}} \cdot \rho_{\mathrm{DM}}^{\frac{5}{3}} \quad$ and $\quad p_{\mathrm{G}}=\kappa_{\mathrm{G}} \cdot \rho_{\mathrm{G}}^{\gamma}$

with the constants $\kappa_{\mathrm{DM}}$ and $\kappa_{\mathrm{G}}$. The initial profiles themselves are computed for hydrostatic equilibrium and its analog for the DM

$$
\frac{\mathrm{d} p_{\mathrm{DM}}}{\mathrm{d} r}=-\rho_{\mathrm{DM}} \frac{\mathrm{d} \Phi}{\mathrm{d} r}, \text { respectively, } \quad \frac{\mathrm{d} p_{\mathrm{G}}}{\mathrm{d} r}=-\rho_{\mathrm{G}} \frac{\mathrm{d} \Phi}{\mathrm{d} r}
$$

Together with the gravitational acceleration $g=-\mathrm{d} \Phi / \mathrm{d} r$ computed by Eq. (3), one gets the following system of equations:

$$
\begin{aligned}
\frac{\mathrm{d}}{\mathrm{d} r} \rho_{\mathrm{DM}} & =\frac{g}{\frac{5}{3} \kappa_{\mathrm{DM}}} \rho_{\mathrm{DM}}^{2-\frac{5}{3}} ; \quad \frac{\mathrm{d}}{\mathrm{d} r} \rho_{\mathrm{G}}=\frac{g}{\gamma \kappa_{\mathrm{G}}} \rho_{\mathrm{G}}^{2-\gamma} \\
\frac{\mathrm{d}}{\mathrm{d} r}\left(r^{2} g\right) & =-4 \pi G\left(\rho_{\mathrm{DM}}+\rho_{\mathrm{G}}\right) r^{2},
\end{aligned}
$$

which can be easily solved by a Runge-Kutta algorithm. The central density of the gas and of the DM, the constants $\kappa_{\mathrm{DM}}$ and $\kappa_{\mathrm{G}}$ introduced above, and the ratio of specific heats for the gas $\gamma$ are free parameters. The central density gradients are set equal to zero. If we want the configuration of both the DM and the gas, to be bound at the same finite radius, then we have to satisfy the condition

$\kappa_{\mathrm{DM}}=\kappa_{\mathrm{G}}\left[\rho_{\mathrm{G}}^{\gamma_{\mathrm{G}}-\frac{5}{3}}\right]_{(r=0)}$.

This eliminates one free parameter. For a better interpretation we choose the halo mass $M_{\mathrm{H}}$, the cut-off radius of the profile $R_{\mathrm{H}}$, and the ratio of specific heats $\gamma$ instead of the parameters introduced above. As an additional constrain we fix the ratio of total DM mass and total gas mass to 0.25 .

As an alternative, we consider the initial DM density distribution realizing an NFW density profile. In that case, the density profile for the baryons is computed as above, but taking the given NFW-profile into account for the DM within the Poisson equation,

$\rho_{\mathrm{NFW}}=\frac{\delta}{r\left(r+r_{\mathrm{s}}\right)^{2}}$.

Here, the normalization for $\delta$ is chosen in a way that the ratio of the total DM mass and the total gas mass is fixed as 0.25 . The characteristic radius is set as an additional parameter. Thus, the equations for the the initial gas profile are

$$
\begin{aligned}
\frac{\mathrm{d}}{\mathrm{d} r} \rho_{\mathrm{G}} & =\frac{g}{\gamma \kappa_{\mathrm{G}}} \rho_{\mathrm{G}}^{2-\gamma} \\
\frac{\mathrm{d}}{\mathrm{d} r}\left(r^{2} g\right) & =-4 \pi G\left(\rho_{\mathrm{NFW}}+\rho_{\mathrm{G}}\right) r^{2} .
\end{aligned}
$$

In a second step, $p_{\mathrm{DM}}$ is determined to keep the system initially in hydrostatic equilibrium (Eq. (12)). 


\subsection{The dynamical evolution}

Once the system is forced to leave the hydrostatic equilibrium, its subsequent evolution is determined by the full timedependent hydrodynamic and Poisson equations. In our case, the subsequent cooling is responsible for this process. In order to compute the evolution of the system, we choose a Lagrangian scheme, which is equivalent to the above Eulerian scheme. This has the benefit of conserving the mass automatically, and the treatment of the outer halo boundary is much simpler. We use the formal analogy discussed above to define a pressure for the $\mathrm{DM}$ as $p_{\mathrm{DM}}=\rho_{\mathrm{DM}} \sigma_{\mathrm{DM}}^{2} / 3$. This allows us to use the same equations for the DM and the gas.

$$
\begin{aligned}
& \frac{\partial \tau}{\partial t}=\frac{\partial}{\partial m}\left(r^{2} u\right) ; \quad \frac{\partial u}{\partial t}=-r^{2} \frac{\partial p}{\partial m}+g \\
& \frac{\partial \varepsilon}{\partial t}=-\frac{\partial}{\partial m}\left(r^{2} u p\right)+u g+\tau \Lambda ; \quad \frac{\partial r}{\partial t}=u \\
& \tau=\rho^{-1} ; \quad \varepsilon=\frac{u^{2}}{2}+\frac{p \tau}{\gamma-1} ; \quad m(r)=\int_{0}^{r} r^{\prime 2} \tau^{-1} \mathrm{~d} r^{\prime} .
\end{aligned}
$$

The gravitation is the only coupling between the two kinds of halo matter, all other calculations may be performed independently for each component using identical routines. The intercell fluxes are computed using a first-order Godunov scheme. We modified the original (second-order) MUSCL scheme presented in van Leer (1979). In our case, due to the high resolution achieved the first-order scheme is sufficient, and encountering strong shocks is not expected. The algorithm is described in detail in Appendix A.

The sources consisting of gravitational acceleration and cooling are calculated in each cell center. The gravitational acceleration at a given radius is computed using the sum of the enclosed DM and gas mass:

$g=-\frac{4 \pi G\left(m_{\mathrm{DM}}+m_{\mathrm{G}}\right)}{r^{2}}$.

The cooling function $\Lambda \tau$ is calculated according to Eqs. (4) and (6).

Instead of the physical quantities, we use a system of dimensionless quantities based on the initial central gas density $\rho_{0}$ and the velocity $u_{0}=c_{0} / \sqrt{\gamma_{\mathrm{G}}}$, with $c_{0}$ the initial central speed of sound of the gas. The dimensionless quantities are computed as $\bar{\rho}=\rho / \rho_{0}, \bar{u}=u / u_{0}, \ldots$ Radius and time are tranformed according to

$r_{0}=\sqrt{u_{0}^{2} /\left(4 \pi G \rho_{0}\right)}, \quad t_{0}=r_{0} / u_{0}=\sqrt{1 /\left(4 \pi G \rho_{0}\right)}$.

The dynamical equations remain unchanged under this transformation, and Eq. (18) simplifies to:

$\hat{g}=-\frac{\left(\hat{m}_{\mathrm{DM}}+\hat{m}_{\mathrm{G}}\right)}{\hat{r}^{2}}$.

Without cooling, the system is freely scalable and the actual dimensions of the halo have no impact on the dynamics. The cooling function has to be computed using the physical density and temperature and must then be transformed according to

$\hat{\Lambda} \hat{\tau}=\frac{\tau_{0} r_{0}}{u_{0}^{2}} \Lambda \tau$

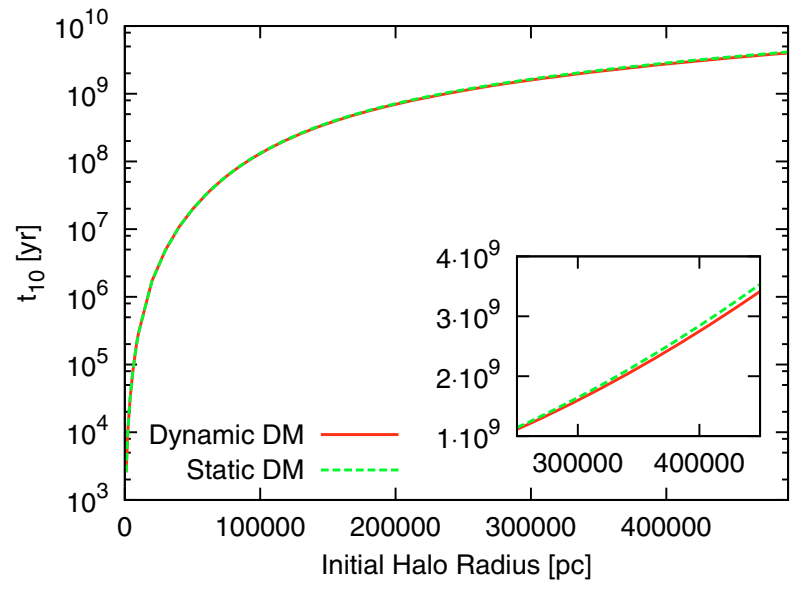

Fig. 1. Time $t_{10}$ when the central gas density.reaches 10 times the initial central density as a function of the initial boundary radii at mass $M_{\mathrm{H}}=$ $10^{11} M_{\odot}$. For fixed mass $R_{\mathrm{H}}$ is a measure for the mean density.

\section{Results}

We performed a series of computations starting with initial stationary configurations as described above. First, it was proven that these configurations (if allowing for their time evolution) are stable against small perturbations as long as no cooling takes place. Then we allowed the gas of the system to cool. This drives the system out of the initial hydrostatic equilibrium. The thermal and dynamical evolution is governed by the system of Eqs. (1), (3), and (9). For halo masses comparable to those of galaxies and more massive objects, the system always collapses within a period comparable with the free fall time of a sphere with mean density $\rho_{\text {average }} \approx M_{\mathrm{H}} / R_{\mathrm{H}}^{3}$. Figure 1 shows a measure of the collapse time in dependence on the initial boundary radii at fixed gas + DM halo mass, i.e. as a function of the mean density.

If the contraction of the DM fraction is taken into account, the collapse time is marginally shorter. We want to get information about the action of the contracting DM onto the stability behaviour of the whole system. Therefore, we consider the behavior of halos with masses of about $M_{6}=10^{6} M_{\odot}$. Those masses are stable against a short cooling period leading to a finite contraction, whereas subsequent cooling also leads to an unlimited collapse. However, as can be seen below, in that case a period of quasi-stability or delayed contraction may occur. This behavior is more sensitive with respect to the dynamics of the DM. In that sense halos with $\approx M_{6}$ represent a limiting case and the action of the co-collapsing DM can be studied in more detail.

To study the influence of the initial density profile, we ran the time-dependent computations for a set of different radii of the initial gas configuration. The NFW profile is formally extending to infinity, but due to the $r^{-3}$ dependence in the outer region it decreases very fast. We located the border of the initial gas sphere well within the region where $\rho_{\mathrm{DM}} \rightarrow 0$ but varied its extension within a relatively narrow range $\left(R_{\mathrm{H}, \mathrm{G}}=[20,28,34] \mathrm{pc}\right)$. For the case of an initially "polytropic" DM distribution we can achieve identical outer boundaries for the DM and gas. In this case we varied the initial radii as $R_{\mathrm{H}, \mathrm{G}}=[16,20,24] \mathrm{pc}$. The particular values for $R_{\mathrm{H}, \mathrm{G}}$ were chosen to demonstrate the possible, qualitatively different results.

Figures 2 and 3 show the typical evolution of the density profiles for gas and DM for an initial NFW profile and a "polytropic" profile (formally with $\gamma_{\mathrm{DM}}=5 / 3$ ) for the DM distribution. When the central gas density increases by 5 orders of 


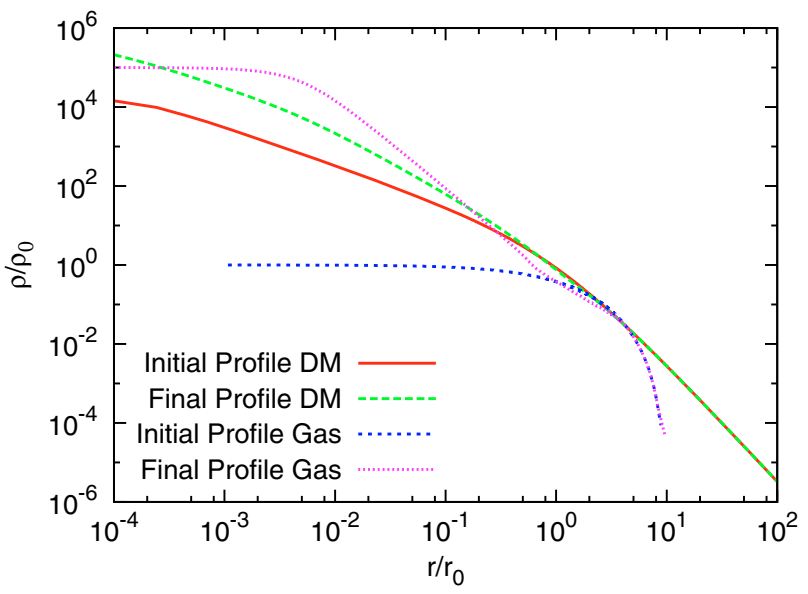

Fig. 2. Initial and final density profiles for DM and gas. The final density profiles are given at the moment when the central gas density reaches the value $10^{5}$. The initial DM profile follows an NFW profile $\left(R_{\mathrm{H}}=\right.$ $20 \mathrm{pc})$.

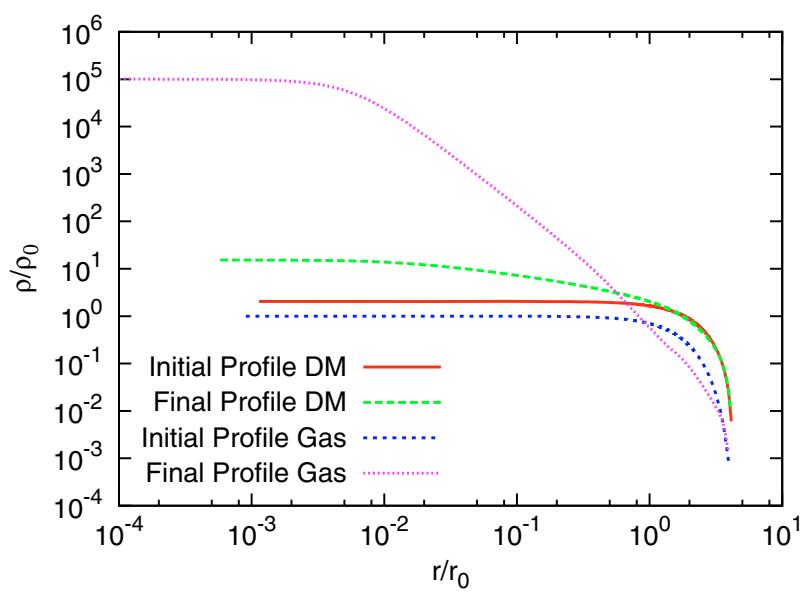

Fig. 3. Same as in Fig. 2 but for an initial "polytropic" DM distribution $\left(R_{\mathrm{H}}=16 \mathrm{pc}\right)$.

magnitude, the central DM density increases by one order of magnitude in both cases. The density profiles steepen with time.

It is obvious that the larger the initial radii, the lower the initial central density. The time evolution of radii containing a fixed amount of mass $(r(0.5 M), r(0.1 M), r(0.01 M))$ is given in Figs. 5 and 6 for NFW and "polytropic" initial DM distributions, respectively.

The time evolution of the central densities shows the most pronounced differences with respect to the chosen initial parameters. Figure 4 shows the time evolution of the central density, the central temperature, and the absolute value of the cooling function for the initial "polytropic" (labeled by A, B, C) and NFW (labeled D, E, F) DM density profiles. In each case three different initial gas density profiles determined by the radii of the halo boundary are considered (see corresponding labels at plots A and D).

The initial boundary radii chosen as small as $\left(R_{\mathrm{H}}=16 \mathrm{pc}\right.$ and $R_{\mathrm{H}}=20 \mathrm{pc}$ ) correspond to steep initial gas profiles. Due to cooling the collapse happens within a relatively short time interval. After an initial period of strong cooling, the cooling function $\Lambda$ (see plots $\mathrm{C}$ and $\mathrm{F}$ ) decreases due to the decline in temperature but rises again catastrophically if the central density increases.
For shallower initial density profiles corresponding to $R_{\mathrm{H}}=$ $20 \mathrm{pc}$ and $R_{\mathrm{H}}=28 \mathrm{pc}$ after a first short contraction phase, an intermediate delayed contraction phase occurs. In this case, the central density is lower when the cooling function rises again. The further increase in density is not enough to compensate for the decline in temperature in order to keep the cooling function increasing. Thus, the cooling function declines before it rises again, like above, and exhibits some hump.

In the case of even shallower profiles $\left(R_{\mathrm{H}}=24 \mathrm{pc}\right.$ and $R_{\mathrm{H}}=34 \mathrm{pc}$ ), the period of delayed contraction is significantly longer (note the logarithmic time scale). Due to the initially lower central density, the cooling function exhibits a series of oscillations until a final unlimited rise in the cooling function occurs.

In all cases, after a very short initial cooling period, the temperature only varies very little during the contraction phase. The oscillations in the plots for the shallowest profile appear as nearly periodic. Figure 8 shows this in a blow-up of the plot during the delayed collapse for two different e.o.s. for the gas $(\gamma=[1.4,1.666])$. The quasi-periodicity depends on the value of $\gamma$ for the gas.

For an interpretation of the behavior described above we refer to Fig. 7. This figure shows the evolution of the characteristic dynamic time $t_{\rho}$, pressure response time $t_{\text {sound }} \propto 1 / \sqrt{T}$ and cooling time $t_{\text {cool }}$ for the intermediate configuration $\left(R_{\mathrm{H}}=20 \mathrm{pc}\right.$, initial "polytropic" DM configuration) in the central region. We notice that after a short cooling period the pressure is not longer able to balance the gravitational force: the dynamical time $t_{\rho}$ becomes shorter than all other characteristic times and the system undergoes contraction without any pressure resistance. The gas falls almost freely. If the initial density is not too high, the collapse gets delayed again, due to the increasing pressure force (the net acceleration becomes less than for pure gravitational infall); however, at nearly constant temperature, the density enhancement due to contraction enforces the cooling process again (the cooling time decreases to a minimum) in such a way that pressure resistance breaks down and the gravitational collaps will be enforced. In this case, this delay happens only once. Then the configuration is driven into the cooling catastrophe; i.e., both the cooling and the dynamical times approach zero. For even shallower initial density profiles, these periods of collapse delay may happen several times.

We want to study the influence of any DM dynamics on the collapse behavior of the gas. To this end we repeated the above calculations under the assumption that the distribution of the DM remains static; i.e., the DM appears as a source of an additional gravitational potential, only. This approximation is often used for only studying the dynamical evolution of the gas embedded in the DM halo (see, e.g., Conroy \& Ostriker 2007).

In Fig. 9 we show the time evolution of the central density in true units for initial NFW DM configurations with $R_{\mathrm{H}}=$ $[20,28,34]$ pc (higher initial central density corresponds to smaller initial $R_{\mathrm{H}}$ ). The upper panel shows the evolution of the DM central densities and the lower panel shows the evolution of central gas densities. The lower panel shows the gas evolution for the case of static DM configuration. Obviously, the evolution goes much faster, if the dynamical evolution of the DM is taken into account. The shallower the initial density profile, the larger the amplification effect appears and the more extended the period of delayed collapse. In Fig. 10 the analogous results for the case of an initial "polytropic" DM configuration is presented $\left(R_{\mathrm{H}}=[20,28,34] \mathrm{pc}\right)$. Note the appearance of the quasioscillations also for the evolution of the central DM density. 


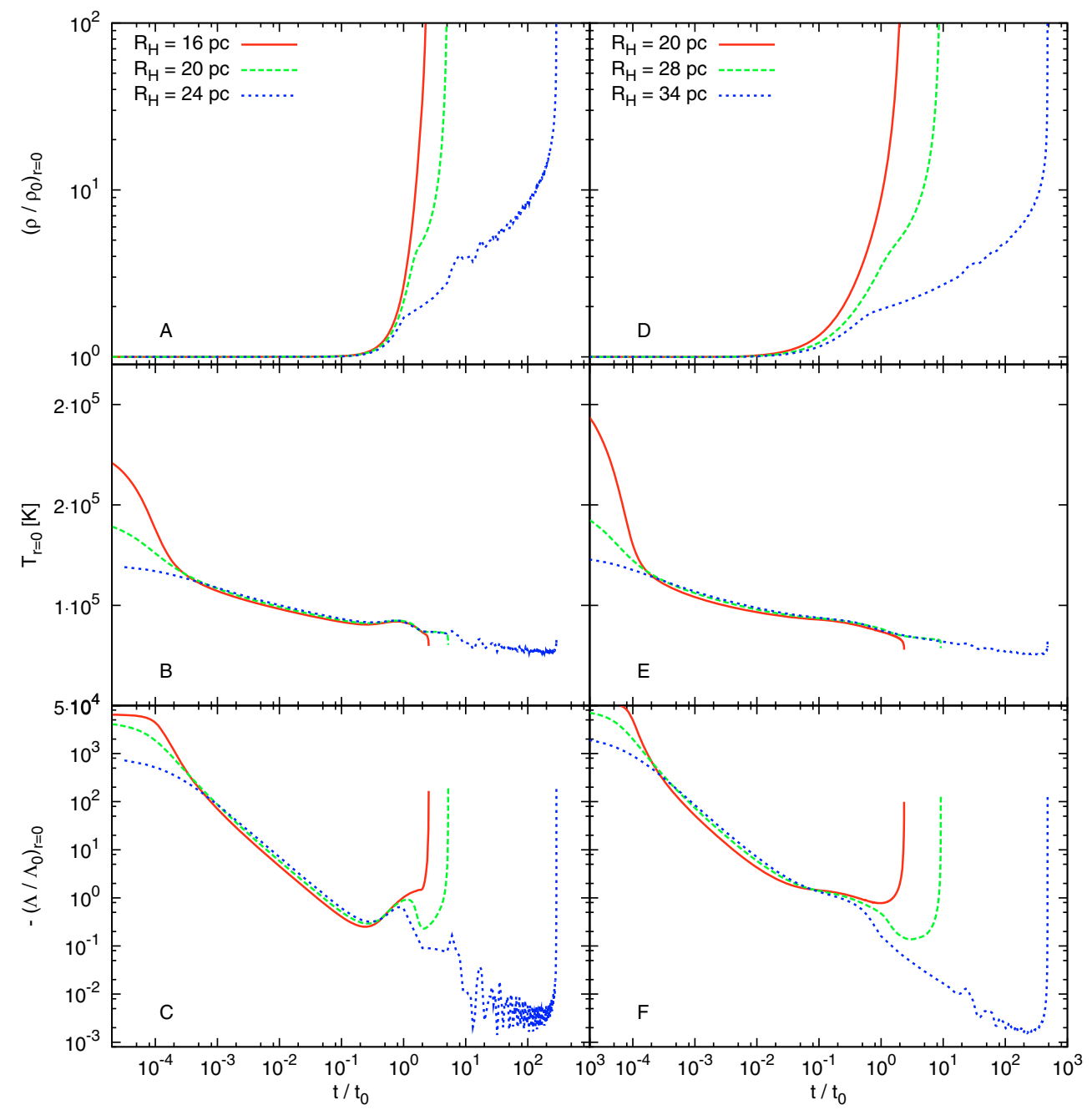

Fig. 4. Time evolution of central gas density (A, D), central temperature (B, E), and of the absolute value of the cooling function (C, F). The left column shows the quantities for the case of an initial "polytropic" DM distribution, the right column shows the behavior for an initial NFW profile of DM distribution.

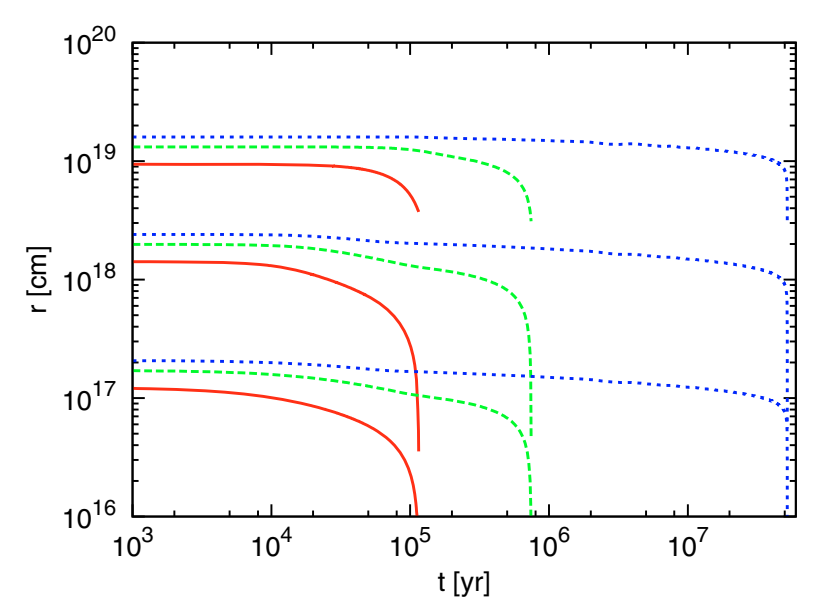

Fig. 5. Time evolution of different radii containing fixed a fraction of mass (top to bottom $r(0.5 M), r(0.1 M), r(0.01 M)$ ) for various initial halo boundaries labeled by line style as in Fig. 4 for an initial NFW profile of the DM.

A measure for the collapse strength could be the period of time $t_{10}$ during which the central density was increasing by a factor of 10 with respect to the initial value $\rho_{0}$. Figure 11 shows the

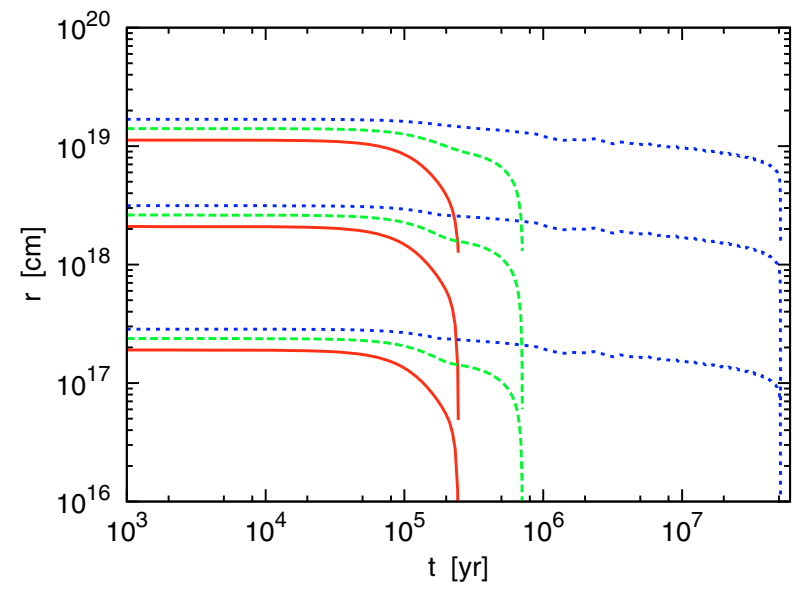

Fig. 6. The same as in Fig. 5 but for an initial "polytropic" DM distribution.

dependence of $t_{10}$ on the chosen initial radii for different polytropic indices in the case of an initial NFW DM density profile. For given mass, the initial radii $R_{\mathrm{H}}$ are a measure for the steepness of the initial density profiles. The line style indicates on the 


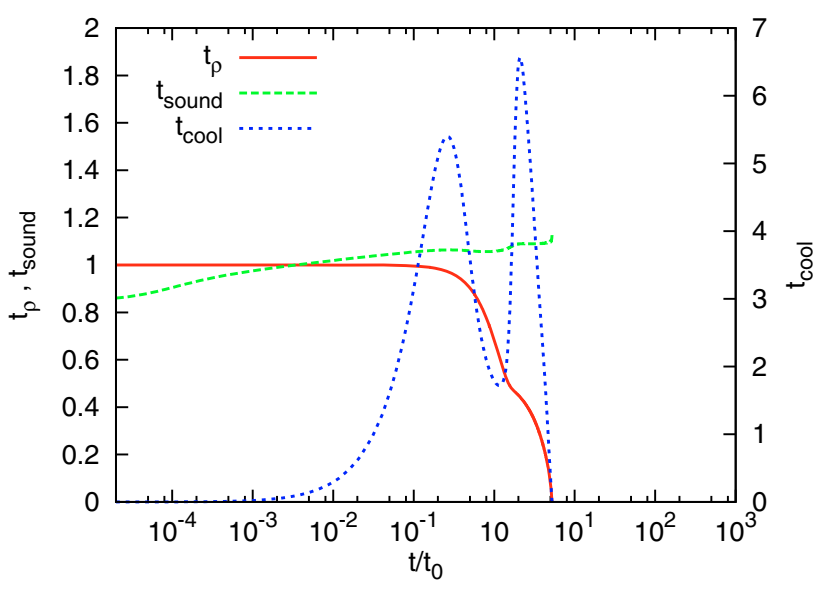

Fig. 7. Time evolution of characteristic dynamic time $t_{\rho}$, pressure response time $t_{\text {sound }} \propto 1 / \sqrt{T}$, and cooling time $t_{\text {cool }}$ for the initial configuration $\left(R_{\mathrm{H}}=20 \mathrm{pc}\right.$, initial "polytropic" DM configuration $)$ in the central region.

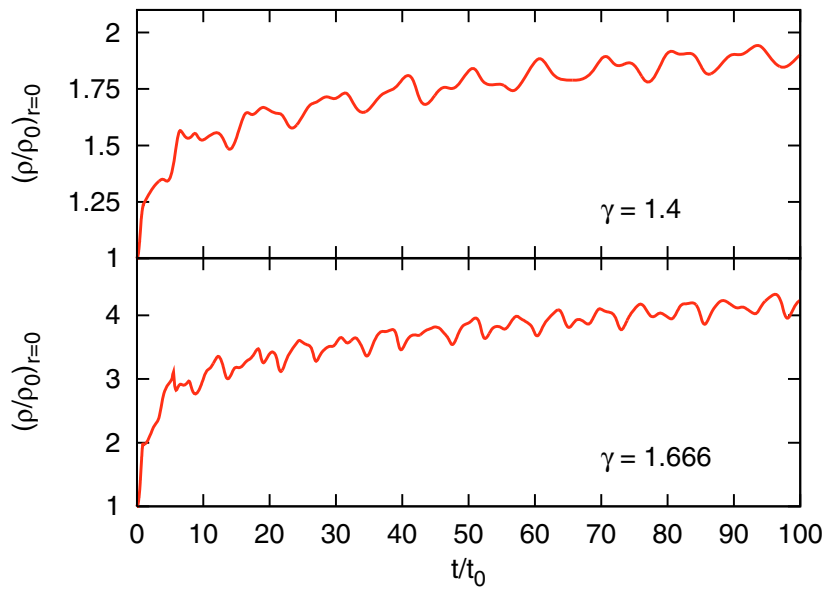

Fig. 8. A blow-up of the time evolution of the central density during the epoch of delayed collapse for two different $\gamma$.

chosen value for $\gamma$, the ratio of specific heats for the gas. The effective $\gamma_{\mathrm{DM}}$ for the DM is fixed to $\gamma_{\mathrm{DM}}=5 / 3$, throughout.

It can be seen that increasing $R_{\mathrm{H}}$ leads to an increasing collapse time. A larger $\gamma$ for the gas, i.e. a stiffer e.o.s. of the gas, leads to a longer $t_{10}$ for given $R_{\mathrm{H}}$. The figure shows pairwiseplotted graphs. In each case, the lefthand-sided graph shows the dependence in case of a static DM distribution. This means that taking a dynamical DM evolution into account coupled to the dynamics of the gas enhances the contraction, i.e. leads to shorter $t_{10}$. One can distinguish three different segments in each graph. Each segment corresponds to a different type of the collapse behavior described above. The transitions between these different collapse phases is characterized by tight intervals in the values $R_{\mathrm{H}}$.

Figure 12 shows the dependences in the case of an initial "polytropic" DM density profile. Here, the static DM distribution leads to much longer $t_{10}$ for given initial radii $R_{\mathrm{H}}$ than in the case of dynamical interaction between gas and DM (labeled by "Dynamic DM" in the plot). Interestingly, the dependence for the dynamic case on the polytropic index is directly opposed to the above case of an initial NFW profile. Here, the three collapse phases seen above degenerate into two, but the transition between them happens within a much narrower range of $R_{\mathrm{H}}$. In the

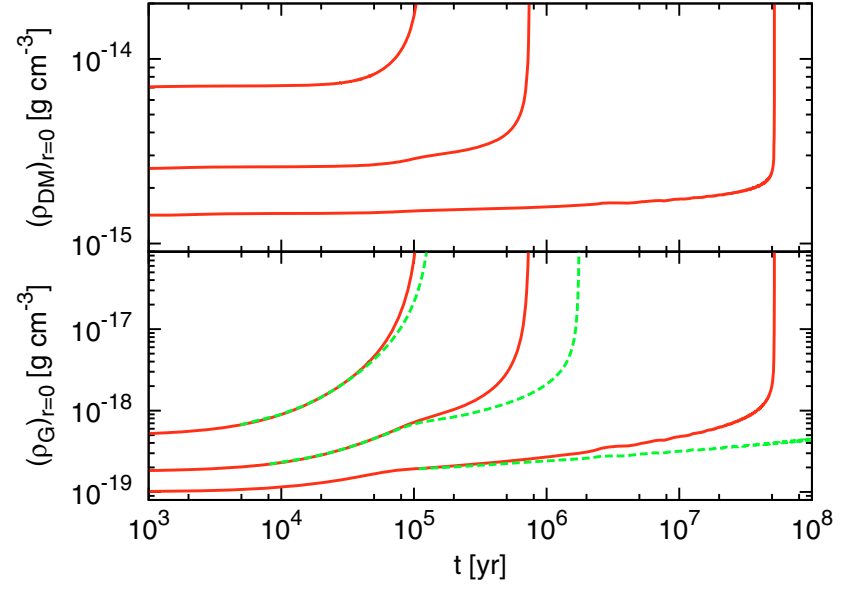

Fig. 9. Time evolution of the central gas and DM density in comparison to the case of an assumed static DM distribution (dashed line) for an initial NFW profile. The true time is given on the $x$-axis.

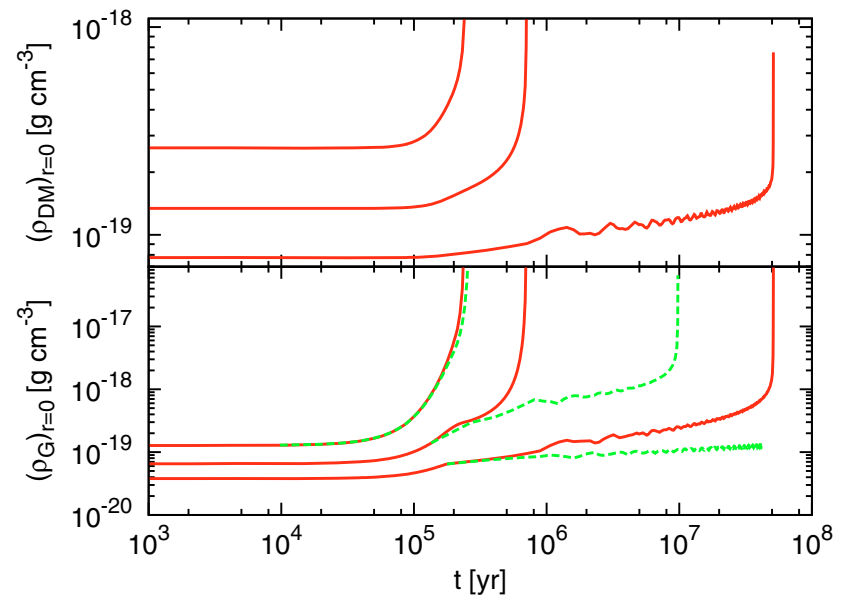

Fig. 10. The same as in Fig. 9 but for an initial "polytropic" DM distribution.

case of the dynamic DM, a break point at radii $R_{\mathrm{H}} \approx 22-23 \mathrm{pc}$ separates the short-time collapse from the delayed collapse.

Whenever the cooling time approaches the dynamical time and both are decreasing fast the cooling catastrophe cannot be avoided. Although we have only considered the example of cooling by recombination, this seems the outcome for all cooling functions $\propto \rho^{2}$ (see also Conroy \& Ostriker 2007); i.e. if the density increases, the cooling time always decreases.

These considerations assume that any dissipated energy can leave the system immediately. If the medium gets opaque enough with respect to interaction of the radiation with matter (e.g. photon-electron scattering), the radiation may need considerable time for carrying away the dissipated energy, which may increase the cooling time considerably. To illustrate that, we introduced a factor $\propto \exp \left(-n \sigma_{T} \lambda\right)$ in front of the cooling function. Here $\lambda$ denotes some characteristic length, $\sigma$ the relevant cross section, and $n$ the number density, e.g., of electrons. At high enough densities $n$ the optical depth $\tau=-n \sigma_{T} \lambda$ may approach unity or may become even greater. In that case the cooling time no longer decreases with increasing density and one could expect that the epoch of delayed collapse may end at a stationary configuration for particular cases. An example is given in Fig. 13 in comparison with the above considered optically thin case. This may indicate a possible self-regulation process for 


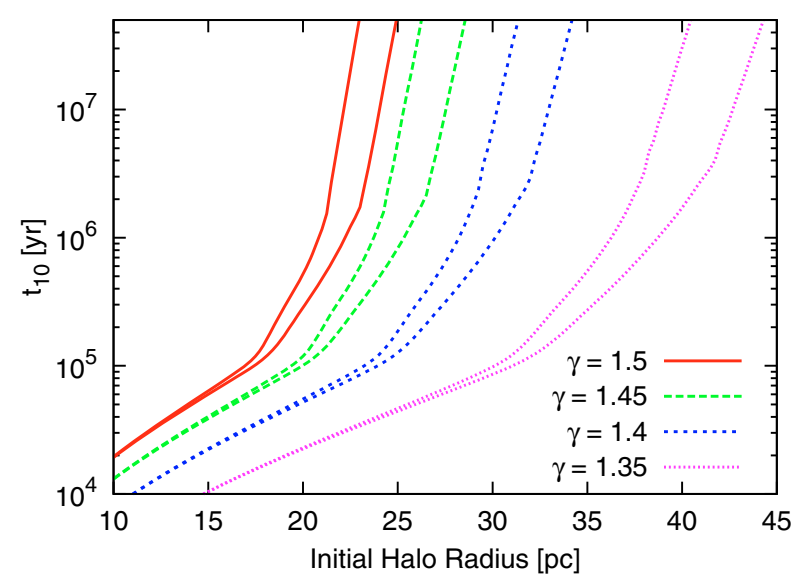

Fig. 11. Collapse time $t_{10}$ defined as the moment when the central gas density $\rho$ reaches $\approx 10 \rho_{\text {ini }}$. Then, $t_{10}$ is shown as function of the initial halo radii (for a fixed mass $M_{\mathrm{H}}=10^{6} M_{\odot}$ ). The initial density for the DM follows the NFW-profile. For each $\gamma$, the left hand graph corresponds to a static DM distribution, while the right hand graph corresponds to the dynamic DM evolution.

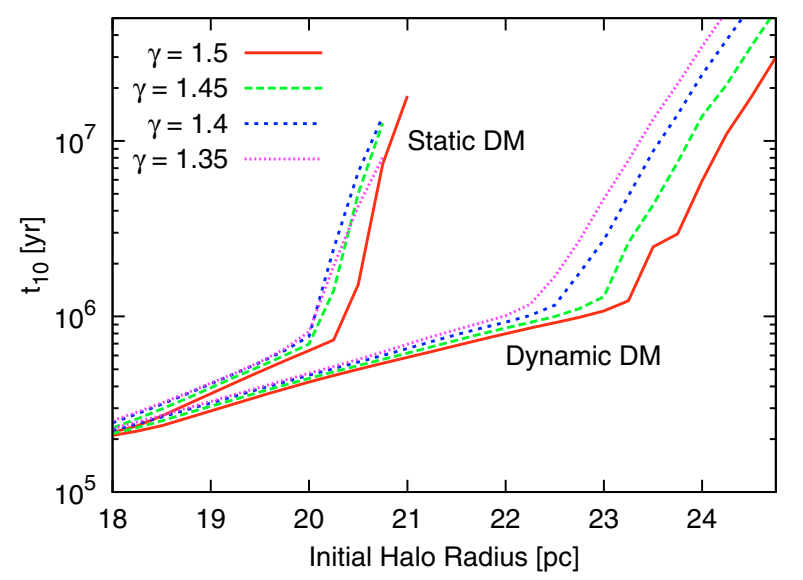

Fig. 12. The same as in Fig. 11 but for initial "polytropic" DM distributions.

halos within a certain mass range. Above all, this concerns dense objects as stars but possibly also the behavior of the very central regions of forming cosmic structures.

\section{Conclusions}

Our goal was to follow the time-dependent behavior of a spherical halo consisting of DM and gas with high accuracy in a 1D model. In particular, we wanted to study the consequences of the dynamic interaction between the two kinds of matter onto the inner matter distribution if the gas is allowed to dissipate energy.

We made the assumption that the DM is dynamically reacting fast enough on any change of the overall gravitational potential via processes of violent relaxation. This justifies our crude fluid approximation for the DM, which actually describes the adiabatic behavior of a collisionless particle configuration under selfgravitation when coupled to an embedded dissipating gas cloud via gravitational interaction alone.

Our results show that the dynamical interaction of gas and DM leads to an enhancement of the collapse behavior of the system (in agreement with the results of Kazantzidis et al. 2006).

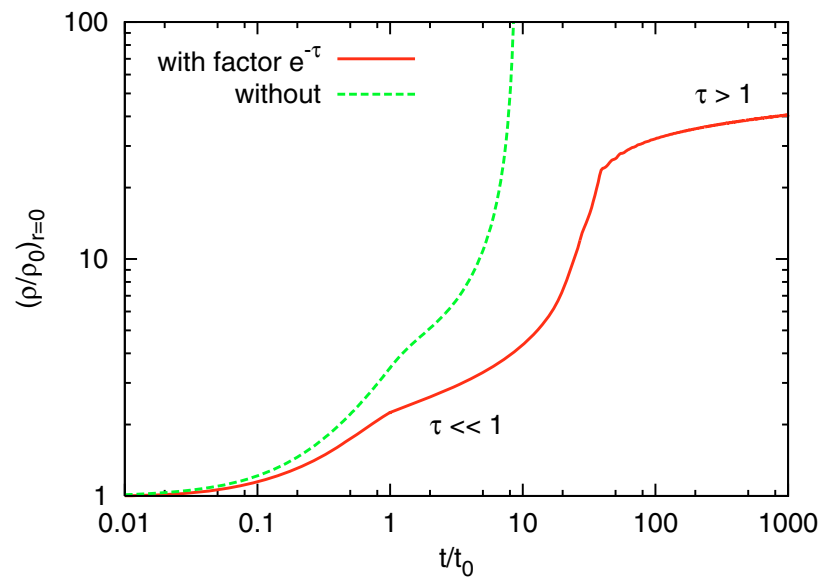

Fig. 13. An example of the modified collapse behavior if the cooling time decreases strongly with increasing optical depth. For comparison, the curve on the left shows the central density evolution for an optically thin medium.

The characteristic times for the contraction process due to the dissipation (cooling) of the gas component are very sensitive to the initial density profiles although the configurations are all initially in a stable hydrostatic equilibrium. The steeper the central density profiles, the shorter the characteristic collapse times. For the halo masses of $\approx M_{6}=10^{6} M_{\odot}$ examined by us, two collapse phases for the gas fraction can be distinguished. After an initial short-period contraction a quasi-stationary state follows, and its duration depends on the initial density profile and possibly on the actual cooling function.

We only considered the processes of collisional ionization and hydrogen recombination cooling. Therefore, the cooling function $\Lambda$ vanishes effectively below $T \approx 10^{4} \mathrm{~K}$. However, molecular hydrogen cooling may be important for halo masses of about $M_{6}=10^{6} M_{\odot}$. Detailed simulations by (Yoshida et al. $2003,2006)$ show that this leads indeed to further gas collapse and eventually to the formation of the first stars in the central region. In this context, the authors stress that processes such as cooling and cloud formation are mainly determined by the particular dynamics of the gravitational collapse. Thus, including molecular hydrogen cooling will lead to an even steeper gas density profile, so it is expected to strengthen the collapse behavior of the whole configuration.

Our result may also enforce the problem described by Conroy \& Ostriker (2007). Their outcome is that only a sensitive fine-tuning of different cooling and heating processes is able to stabilize configurations like clusters over a time comparable with the Hubble time, at least. Taking into account the dynamical interaction between thermo-dynamically evolving gas and the DM halo, such a fine-tuning may be difficult to adjust. Especially within the central region of the considered configurations, the dynamic interaction between gas and DM may alter the situation considerably.

Our computations do not indicate any situation where the innermost DM distribution might get shallower due to interaction with the gas. Thus, self-consistently including the gravitational interaction between DM and gas is not yet enough to solve the discrepancy between the NFW density profile for DM halos in simulations and the indications on a core distribution from observed rotational curves of galaxies. The tendency of even steepening the inner DM profile by gas cooling may influence the investigations of DM annihilation signals. 
Our results demonstrate that the gravitational interaction between gas and DM may have a strong influence on the dynamical evolution of the halo systems during cosmic evolution. We have not included the possibility of continuous matter infall/accretion onto the evolving gas-DM halos. Further on, we did not include non-isotropic velocity dispersions and a non-vanishing angular momentum. This may alter the situation (see, e.g., recent work by Tonini et al. 2006), so it will be the subject of forthcoming research.

Acknowledgements. We thank Naoki Yoshida for giving us many constructive comments and helpful suggestions. J.K. was supported by the Deutsche Forschungsgemeindschaft under the project MU 1020/6-4.s.

\section{Appendix A: Numerical algorithms}

In the first step, velocity $u^{*}$ and pressure $p^{*}$ at the cell interfaces are determined by an exact Riemann solver. With these values the position of the cell interfaces after half the timesteps are computed:

$r_{i+\frac{1}{2}}^{\prime}=r_{i+\frac{1}{2}}(t)+\frac{1}{2} \Delta t u_{i+\frac{1}{2}}^{*}$.

Because the mass coordinates of DM and gas are independent, the gravitational acceleration for the DM is computed by interpolating the enclosed mass of the gas using the trapezoid rule:

$\left[g_{\mathrm{DM}}\right]_{i}=4 \pi G \frac{\left[m_{\mathrm{DM}}\right]_{i}+\left[m_{\mathrm{G}}\right]_{i}}{\bar{r}_{i}^{2}}$

with:

$\bar{r}_{i}=\frac{\left[r_{\mathrm{DM}}^{\prime}\right]_{i+\frac{1}{2}}+\left[r_{\mathrm{DM}}^{\prime}\right]_{i-\frac{1}{2}}}{2}$

$\left[m_{\mathrm{DM}}\right]_{i}=\frac{\left[m_{\mathrm{DM}}\right]_{i+\frac{1}{2}}+\left[m_{\mathrm{DM}}\right]_{i-\frac{1}{2}}}{2}$

$\left[m_{\mathrm{G}}\right]_{i}=\left[m_{\mathrm{G}}\right]_{j+\frac{1}{2}}+\left(\bar{r}-\left[r_{\mathrm{G}}^{\prime}\right]_{j+\frac{1}{2}}\right) \frac{\left[m_{\mathrm{G}}\right]_{j+\frac{3}{2}}-\left[m_{\mathrm{G}}\right]_{j+\frac{1}{2}}}{\left[r_{\mathrm{G}}^{\prime}\right]_{j+\frac{3}{2}}-\left[r_{\mathrm{G}}^{\prime}\right]_{j+\frac{1}{2}}}$

with the gas cell $j$, the outermost gas cell enclosed $\left(\left[\bar{r}_{\mathrm{DM}}\right]_{i}>\right.$ $\left.\left[r_{\mathrm{G}}^{\prime}\right]_{j+\frac{1}{2}}>\left[\bar{r}_{\mathrm{DM}}\right]_{i-1}\right)$. The procedure for calculating the gravitational acceleration for the gas is analogous. For computation of the cooling function, the temperature is computed according to

$T_{i}=\frac{m_{\mathrm{H}}}{k_{\mathrm{B}}} p_{i} \tau_{i}$.

Then, the cooling function $[\Lambda \tau]_{i}$ can be calculated using Eqs. (4), (6) and (21). To conclude the timestep, the quantities at the time $t+\Delta t$ are now computed (in this order):

$$
\begin{aligned}
r_{i+\frac{1}{2}}(t+\Delta t)= & r_{i+\frac{1}{2}}(t)+u_{i+\frac{1}{2}}^{*} \cdot \Delta t \\
\tau_{i}(t+\Delta t)= & \frac{r_{i+\frac{1}{2}}^{3}}{3}-\frac{r_{i-\frac{1}{2}}^{3}}{3} \\
u_{i}(t+\Delta t)= & u_{i}(t)-\frac{\Delta t}{\Delta m_{i}}\left[\left[r^{\prime 2} p^{*}\right]_{i+\frac{1}{2}}-\left[r^{\prime 2} p^{*}\right]_{i-\frac{1}{2}}\right. \\
& \left.-\frac{p_{i+\frac{1}{2}}^{*}+p_{i-\frac{1}{2}}^{*}}{2}\left(r^{\prime} 2_{i+\frac{1}{2}}-r^{\prime} 2_{i-\frac{1}{2}}\right)\right]+\Delta t g_{i} \\
\varepsilon_{i}(t+\Delta t)= & \varepsilon_{i}(t)-\frac{\Delta t}{\Delta m_{i}}\left[\left[r^{\prime 2} p^{*} u^{*}\right]_{i+\frac{1}{2}}-\left[r^{\prime 2} p^{*} u^{*}\right]_{i-\frac{1}{2}}\right] \\
& +\Delta t\left[\frac{u_{i+\frac{1}{2}}^{*}+u_{i-\frac{1}{2}}^{*}}{2} g_{i}+[\Lambda \tau]_{i}\right]
\end{aligned}
$$

The boundary condition at $r=0$ is realized by a reflecting boundary. Central pressure and velocity are computed using the solution of the Riemann-problem between the innermost cell and a ghost cell with the same density and pressure, but with a negative velocity. The boundary between gas and vacuum is treated by solving the vacuum Riemann problem (see Toro 1999 , p. 138). The solution only consists of one rarefaction wave ending at the contact discontinuity. Thus, the pressure is $p^{\star}=0$ there. Using the Lagrangian version of the generalized Riemann invariant (see van Leer 1979, p. 105),

$\left(u^{\star}-u\right) \mp \frac{2}{\gamma-1}\left(\tau^{\star} a^{\star}-\tau a\right)=0$,

one gets for the velocity

$u^{\star}=u_{i_{\max }}+\frac{2}{\gamma-1} \tau_{i_{\max }} a_{i_{\max }}$

with the Lagrangian speed of sound $a=\sqrt{\gamma p / \tau}$.

Instead of a sharp cut-off, the NFW-profile only reaches zero for $r \rightarrow \infty$. We use a reflecting boundary condition there to inhibit any artificial inflow or outflow. Since the outer boundary of the DM profile is much more extended than for the gas, this choice should not affect the dynamics of the central region.

\section{References}

Ascasibar, Y., Jean, P., Boehm, C., \& Knödlseder, J. 2006, MNRAS, 368, 1695 Binney, J., \& Tremaine, S. 1987, Galactic Dynamics (Princeton University Press) Blumenthal, G. R., Faber, S. M., Flores, R., \& Primack, J. R. 1986, ApJ, 301, 27 Black, J. H. 1981, MNRAS, 197, 553

Cardone, V. F., \& Sereno, M. 2005, A\&A, 438, 545

Chièze, J.-P., Teyssier, R., \& Alimi, J.-M. 1997, ApJ, 484, 40

Conroy, C., \& Ostriker, J. P. 2007 [arXiv: astro-ph/07120824]

Diemand, J., Kuhlen, M., \& Madau, P. 2007, ApJ, 657, 262

Dutton, A. A., van den Bosch, F. C., Dekel, A., Courteau, S., \& Cardone, V. F. 2007, ApJ, 654, 27

Eggen, O. J., Lynden-Bell, D., \& Sandage, A. R. 1962, ApJ, 136, 748

Gentile, G., Salucci, P., Klein, U., Vergani, D., \& Kalberla, P. 2004, MNRAS, 351,903

Gnedin, O. Y., Kravtsov, A. V., Klypin, A. A., \& Nagai, D. 2004, ApJ, 616, 16

Hoeft, M., Mücket, J. P., \& Gottlöber, S. 2004, ApJ, 602, 162

Jesseit, R., Naab, T., \& Burkert, A. 2002, ApJ, 571, L89

Kazantzidis, S., Kravtsov, A. V., Zentner A. R., et al. 2004, ApJ, 611, L73

Kleinheinrich, M., Schneider, P., Rix, H.-W., et al. 2006, A\&A, 455, 441

Kull, A., Treumann, R. A., \& Böringer, H. 1997, ApJ, 484, 58

Kuzio de Naray1, R., McGaugh1, S. S., de Blok1, W. J. G., \& Bosma, A. 2006, ApJS, 165, 461

van Leer, B. 1997, J. Comp. Phys., 32, 101

Lyndon-Bell, D. 1967, MNRAS, 136, 101

Marchesini, D., D’Onghia, E., Chincarini, G., et al. 2002, ApJ, 575, 801

Navarro, J. F., Frenk, C. S., \& White, S. D. M. 1997, ApJ, 490, 493

Ryden, B. S., \& Gunn, J. E. 1987, ApJ, 318, 15

Persic, M., Salucci, P., \& Stel, F. 1996, MNRAS, 281, 27

Ryden, B. S., \& Gunn, J. E. 1987, ApJ, 318, 15

Salucci, P., Lapi, A., Tonini, C., et al. 2007, MNRAS, 378, 41

Sellwood, J. A., \& McGaugh, S. S. 2005, ApJ, 634, 70

Stoehr, F., White, S. D. M., Springel, V., Tormen, G., \& Yoshida, N. 2003, MNRAS, 345, 1313

Taylor, J. E., \& Navarro, J. F. 2001, ApJ, 563, 483

Teyssier, R., Chièze, J.-P., \& Alimi, J.-M. 1997, ApJ, 484, 36

Toro, E. 1999, Riemann Solvers and Numerical Methods for Fluid Dynamics, 2nd edition (Berlin Heidelberg: Springer-Verlag)

Tonini, C., Lapi, A., \& Salucci, P. 2006, 649, 591

Vasiliev, E. 2006, JETO Letters, 84/2 [arXiv: astro-ph/0601669]

Yoshida, N., Abel, T., Hernquist, L., \& Sugiyama, N. 2003, ApJ, 592, 645

Yoshida, N., Kazuyuki, O., Hernquist, L., \& Abel, T. 2006, ApJ, 652, 6

Young, P. 1980, ApJ, 242, 1232

Zeldovich, Y. B., Klypin, A. A., Khlopov, M. Y., \& Chechetkin, V. M. 1980, Soviet J. Nucl. Phys., 31, 664 